This item was submitted to Loughborough's Research Repository by the author.

Items in Figshare are protected by copyright, with all rights reserved, unless otherwise indicated.

\title{
A theoretical analysis of billiard ball dynamics under cushion impacts
}

PLEASE CITE THE PUBLISHED VERSION

http://dx.doi.org/10.1243/09544062JMES1964

PUBLISHER

Sage Publications / @ The Authors

\section{VERSION}

SMUR (Submitted Manuscript Under Review)

\section{LICENCE}

CC BY-NC-ND 4.0

\section{REPOSITORY RECORD}

Mathavan, Senthan, Michael R. Jackson, and Robert M. Parkin. 2019. "A Theoretical Analysis of Billiard Ball Dynamics Under Cushion Impacts”. figshare. https://hdl.handle.net/2134/15087. 
This item was submitted to Loughborough's Institutional Repository (https://dspace.lboro.ac.uk/) by the author and is made available under the following Creative Commons Licence conditions.

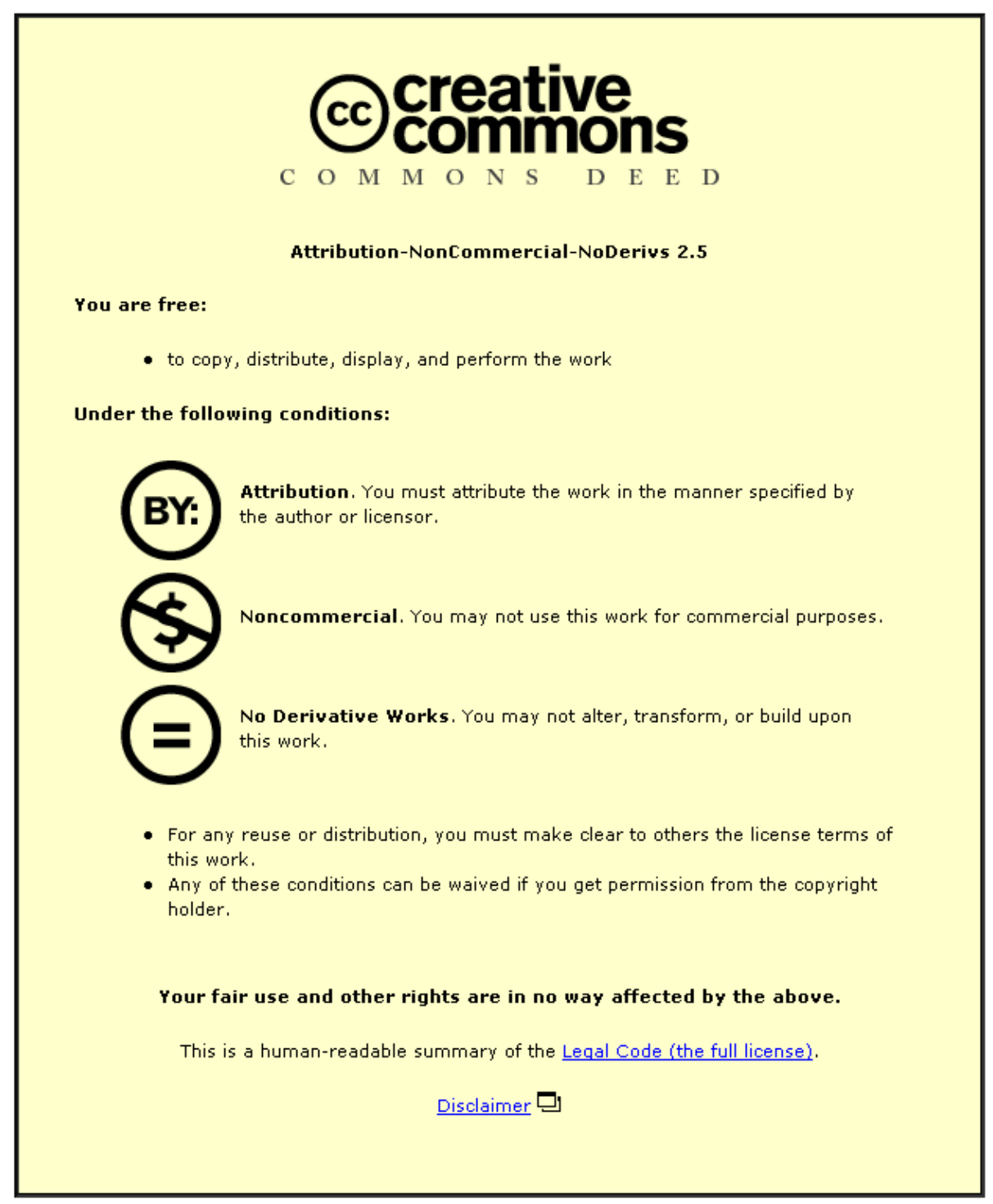

For the full text of this licence, please go to: http://creativecommons.org/licenses/by-nc-nd/2.5/ 


\title{
A theoretical analysis of billiard ball dynamics under
}

\section{cushion impacts}

\author{
S Mathavan*, M R Jackson, and R M Parkin \\ Mechatronics Research Group, Wolfson School of Mechanical and Manufacturing \\ Engineering, Loughborough University, Loughborough, LE11 3UZ, UK
}

\begin{abstract}
In the last two decades there has been a growing interest in research related to billiards. There have been a number of projects aimed at developing training systems, robots and computer simulations for billiards. The trajectory of the billiard ball is important for all these systems mentioned. The ball-cushion collision is often encountered in billiards and it drastically changes the ball trajectory, especially when the ball has spin. This work predicts the off-thecushion bounce angles and speeds, under the assumption of insignificant cushion deformation. Differential equations are derived for the ball dynamics during impact and are solved numerically. The numerical solutions together with a previous experimental work by the authors predict that the coefficient of restitution between the ball and cushion is 0.98 . Also the sliding coefficient of friction between the ball and cushion is found to be 0.14 . A comparison of numerical and experimental results indicates that the limiting normal velocity under which the rigid cushion assumption is valid is $2.5 \mathrm{~m} / \mathrm{s}$, which in billiards is above average. A number of plots that shows the rebound characteristics for given velocity-spin conditions are given at the end. The plots quantify various phenomena that are only explained in billiards literature hitherto.
\end{abstract}

Keywords: impulse with friction, billiards, snooker, pool, ball trajectories, cushion rebound, coefficient of restitution 


\section{INTRODUCTION}

Snooker and pool are two popular cue sports generally known as billiards (here onwards the term billiards is used to refer to both snooker and pool together). Billiards is one of the first games to be analysed from a technical perspective. The 1835 study of famous French scientist Coriolis, named Théorie mathématique des effets du jeu de billard, is a pioneering work on sports dynamics [1]. Billiards is a classic example of dynamic concepts such as spinning, rolling, sliding and collisions of spheres. In recent years there is a growing interest on billiards related research. A number of robots have been developed to play pool and snooker $[\mathbf{2}, \mathbf{3}, \mathbf{4}, \mathbf{5}]$. There are also a few initiatives on creating training systems for billiard games $[\mathbf{6}$, 7]. The research on computer billiards, which simulates the real-world billiards environment, also receives an increasing attention from computer scientists in creating artificial intelligence to formulate appropriate game playing strategies $[\mathbf{8 , 9}, \mathbf{1 0}]$.

Billiards is about accurately manipulating the balls on the table along different trajectories. This is performed so that all object balls are potted, in the given order, and the cue ball is left at an advantageous position on the table, after each shot, to play the next shot successfully. A player often uses cushion (or wall/rail/bumper) impacts to achieve the planned trajectories. Cushion impacts give a great deal of variation to the game. Ball-cushion impact, combined with the effects of ball spin, changes the ball trajectories dramatically, and provides the player a greater flexibility in his game strategy (see Fig. 1). 


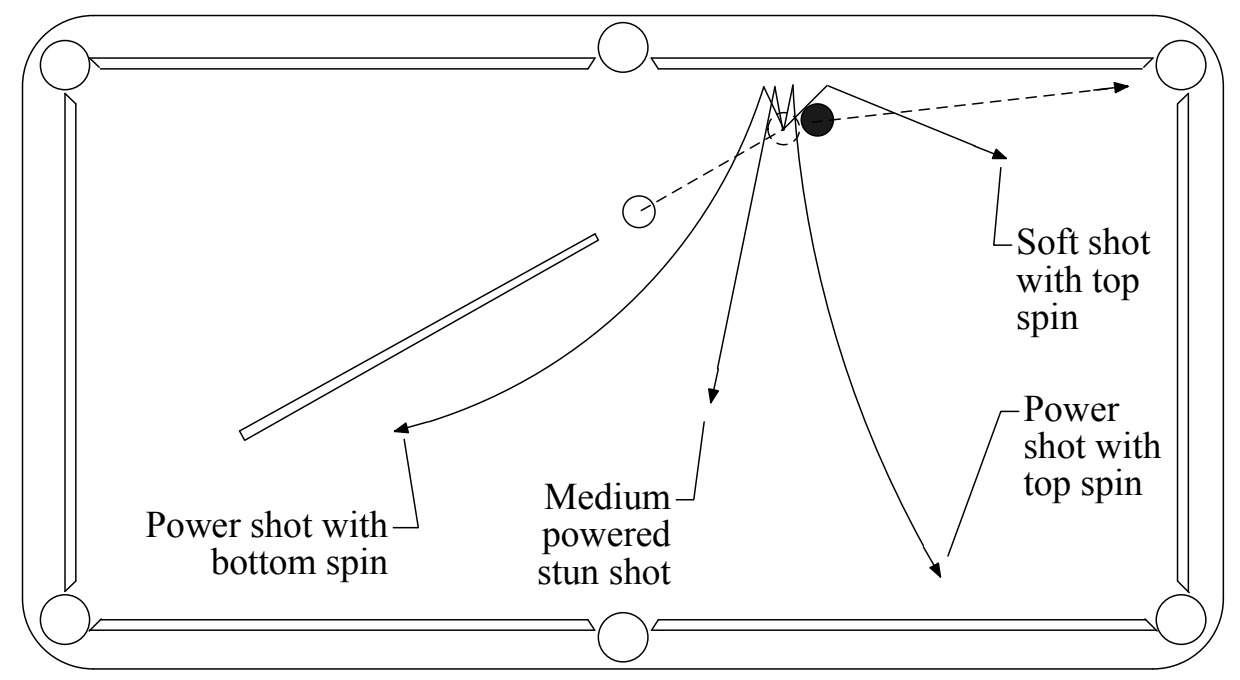

Fig. 1 Positioning the cue ball by its bounce off the cushion, by imparting it with different ball spins, still potting the object ball (shown in black)

Up to now the ball bounces off the cushion are analysed incorporating the coefficient of restitution, between the ball and cushion, as the only influencing parameter. In this analysis, the ball velocity normal to the cushion is considered as the sole variable. According to this theory, referring to Fig. 2, once the ball bounces off the cushion, it will have a velocity of $e_{e} V_{0} \sin \alpha$ and $V_{0} \cos \alpha$ along the normal, and the directions to the cushion respectively, where $e_{e}$ is the coefficient of restitution between the ball and cushion. The analysis does not take the effects of ball spin and the effect of friction during the impact into account, and purely treats it as a two dimensional phenomenon (the plane of analysis is as given in Fig. 2). However the spin on the ball, both sidespin $\omega^{S}$ and topspin $\omega^{T}{ }_{0}$ as shown in Fig. 2, are known to affect both the rebound speed and rebound angle $\beta$ of the ball. The latter two quantities are vital in estimating the trajectory of the ball off the cushion. Even though Marlow [11] has tried to address these issues, the approach of the analysis involves unnecessary parameters like the impact time between the cushion and ball for which the values were not known. Other assumptions such as taking sliding directions as constant do not seem correct (it is shown later that these keep changing, throughout impact). 
In this paper a 3-dimensional analysis of the cushion-ball impact, is presented. For a given input conditions the analysis enables the calculation of rebound conditions. This work will be useful for robotics research on billiards that involves trajectory calculations for the ball motion. The trajectory estimation is also necessary for the systems that train amateur billiard players, as they need to instruct the player how a given shot (with a given speed and spin) will change the configuration of the balls on the table. Also the computer simulations, when incorporated with the knowledge from this 3 dimensional impact analysis, would give the user a more realistic experience of the game. This work will also be of interest to the researchers that work on the physics of billiards (for an exhaustive list of publications on billiard physics see Alciatore [11]).

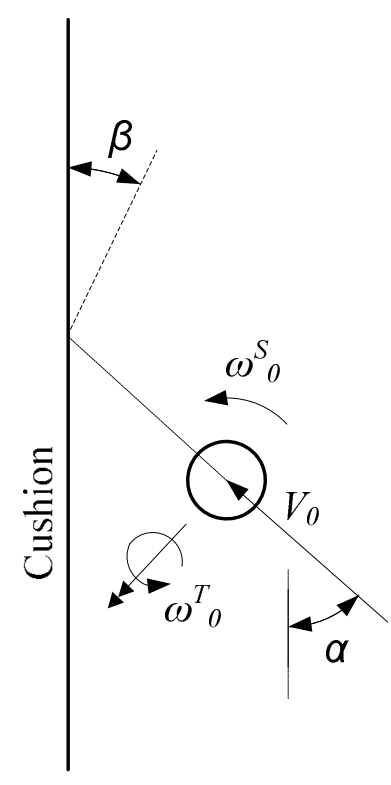

Fig. 2 Billiard ball prior to collision with a cushion

\section{THEORY}

The billiards cushion is made out of pure gum rubber that has good rebound properties. The cross section of a typical billiard cushion is shown in Fig. 3. Usually a slope is provided in the 
cushion such that its contact point on the ball is always above the horizontal great circle of the ball, in order to prevent the ball from leaping up in the air after impact. Here, the cushion is assumed not to change its geometry during the impact with the ball. This assumption may not be valid at high ball speeds, as the normal ball velocity at the contact point, I (see Fig. 3), will deform the tip of the cushion. Also a point contact is always assumed between the ball and cushion, which again may not be true at high ball speeds, as the ball will start to 'sink' more into the rubber cushion.

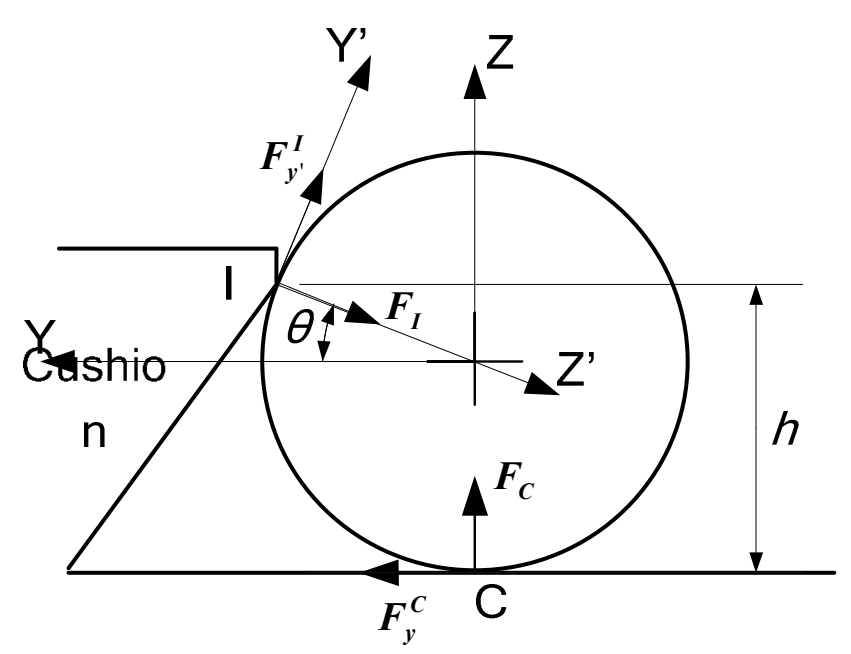

Fig. 3 The forces acting on the ball at the moment of collision - a side view along the cushion at the table level

In Fig. 2, the height of the contact point at the rail (i.e. I) is $h$. In both snooker and pool $h=7 R / 5$, where $\mathrm{R}$ is ball radius. At the contact point with the cushion, the common normal line $Z^{\prime}$ makes an angle of $\theta$ with the $Y$ axis. Thus, $\sin \theta=\frac{2}{5}$. 


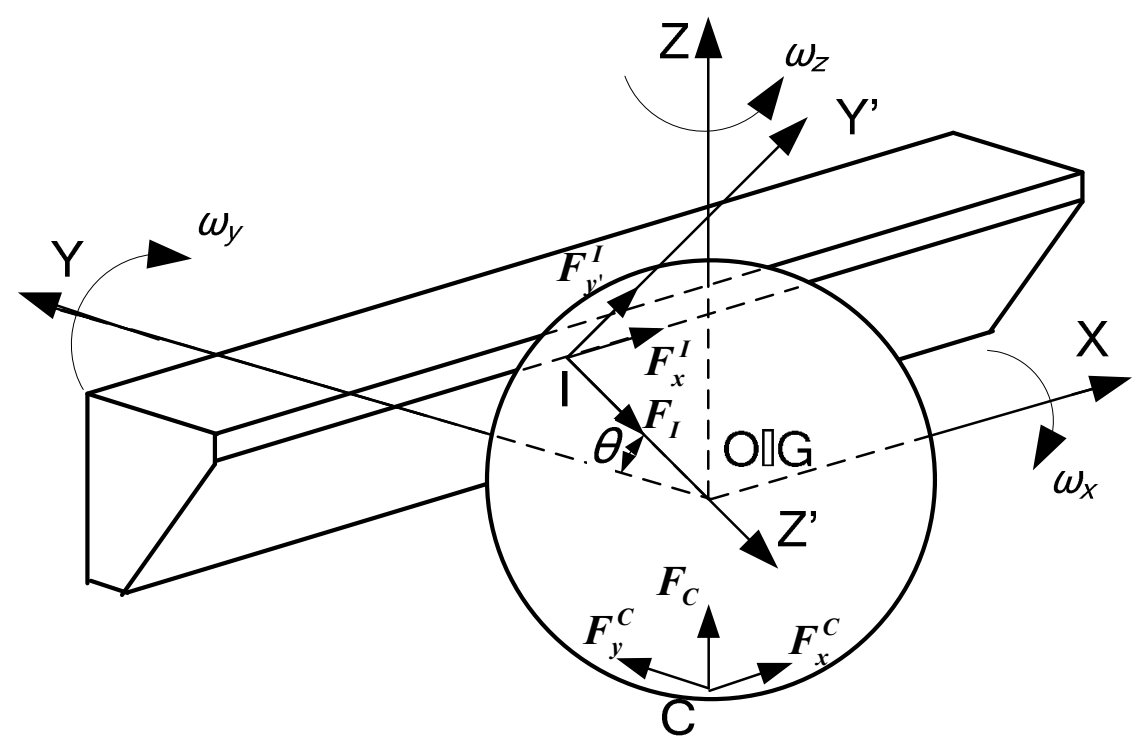

Fig. 4 The forces on the ball during impact (a part of the cushion is shown)

\subsection{General equations of motion for the ball}

Referring to Fig. 3, for the ball, along X, Y and Z directions respectively,

$F_{x}^{I}+F_{x}^{C}=M \ddot{x}_{G}$

$-F_{I} \cos \theta-F_{y^{\prime}}^{I} \sin \theta+F_{y}^{C}=M \ddot{y}_{G}$

$-F_{I} \sin \theta+F_{y^{\prime}}^{I} \cos \theta+F_{C}-M g=M \ddot{z}_{G}$

During the period of impulse, at any time instant $t$, consider a time period of $\Delta t$. Now, let $\Delta P$ denote the impulse due to the action of a general force $F$ over $\Delta t$. Also the accumulated total impact up to time $\mathrm{t}$ is denoted as $P$ (and assuming the impact started at $t=0$ ), now, it could be written that,

$\Delta P=\int_{t}^{t+\Delta t} F \cdot d t$

and, 


$$
P=\sum \Delta P=\int_{0}^{t} F \cdot d t
$$

The impulse-momentum relationship in conjunction with (2a), along the above directions results in the following equations.

$\Delta P_{x}^{I}+\Delta P_{x}^{C}=M \Delta \dot{x}_{G}$

$-\Delta P_{I} \cos \theta-\Delta P_{y^{\prime}}^{I} \sin \theta+\Delta P_{y}^{C}=M \Delta \dot{y}_{G}$

$-\Delta P_{I} \sin \theta+\Delta P_{y^{\prime}}^{I} \cos \theta+\Delta P_{C}=M \Delta \dot{z}_{G}$

In equation (3c), it should be noted that the impact component due to the mass of the ball $m g$ is absent. According to de la Juarez [12], in the limit $\Delta t \rightarrow 0$, the non-diverging forces, such as the weight $m g$, will have a negligible contribution, thus will not influence the increase in momentum. In (3c) it should also be noted that due to the physical sloped shape of the cushion the vertical motion of the ball is constrained. Hence, $\Delta \dot{z}_{G}=0$. Equation (3c) could be rearranged as,

$$
\Delta P_{C}=\Delta P_{I} \sin \theta-\Delta P_{y^{\prime}}^{I} \cos \theta
$$

Similarly for the rotational motion of the ball about the $\mathrm{X}$ axis, the following equation could be derived, with angular velocity being denoted by $\omega$, $\left(\Delta P_{y^{\prime}}^{I}+\Delta P_{y}^{C}\right) R=I \Delta \omega_{x}$

Where, the moment of inertia of the ball, $I=\frac{2 M R^{2}}{5}$. The above equation could be written as,

$$
\Delta P_{y^{\prime}}^{I}+\Delta P_{y}^{C}=\frac{2 M R}{5} \Delta \omega_{x}
$$

Similarly about $\mathrm{Y}$ and $\mathrm{Z}$ axes,

$$
\begin{aligned}
& \Delta P_{x}^{I} \sin \theta-\Delta P_{x}^{C}=\frac{2 M R}{5} \Delta \omega_{y} \\
& -\Delta P_{x}^{I} \cos \theta=\frac{2 M R}{5} \Delta \omega_{z}
\end{aligned}
$$




\subsection{Impact dynamics at points I and C}

At the contact point with the cushion, I, generally it could be said that the ball is slipping on the rail (the rolling could be treated as a special case of slipping, where the slipping velocity is zero). The slip will take place on the XY' plane (i.e. the tangential plane), also noting that the axis $\mathrm{Y}^{\prime}$ is in the $\mathrm{YZ}$ plane. Let the slip speed of the ball at I, be $s(t)$ at an angle $\Phi(t)$ from the $\mathrm{X}$ axis. The instantaneous value of the normal impulse $P_{I}$ according to equation (2b) will always be positive as $F_{I}$ is always positive. $P_{I}$ monotonously increases with time $t$ within the interval of impact. Hence generally it is taken as an independent variable, instead of time $t$ [13]. See Stronge [13] for an elaborative explanation on this and on other principles used within the subsection 2.2. The slipping velocities along $\mathrm{X}$ and $\mathrm{Y}^{\prime}$ axes are given by, respectively,

$$
\begin{aligned}
& \dot{x}_{I}=s\left(P_{I}\right) \cos \left(\Phi\left(P_{I}\right)\right) \\
& \dot{y}_{I}^{\prime}=s\left(P_{I}\right) \sin \left(\Phi\left(P_{I}\right)\right)
\end{aligned}
$$

But $\dot{y}_{I}^{\prime}$ could be written as,

$$
\dot{y}_{I}^{\prime}=-\dot{y}_{I} \sin \theta+\dot{z}_{I} \cos \theta
$$

Using the Amontons-Coulomb law of friction, for $s>0$, also noting that the friction forces/impulses are opposite to the direction of sliding, friction impulses along $\mathrm{X}$ and $\mathrm{Y}^{\prime}$ are

$$
\begin{aligned}
& \Delta P_{x}^{I}=-\mu_{w} \cos \left(\Phi\left(P_{I}\right)\right) \Delta P_{I} \\
& \Delta P_{y^{\prime}}^{I}=-\mu_{w} \sin \left(\Phi\left(P_{I}\right)\right) \Delta P_{I}
\end{aligned}
$$

Where, $\mu_{w}$ is the coefficient of friction between the ball and cushion.

From (3d) and (7b), the normal reaction from the table surface to the ball is given by,

$$
\Delta P_{C}=\left(\sin \theta+\mu_{w} \sin \left(\Phi\left(P_{I}\right)\right) \cos \theta\right) \Delta P_{I}
$$

Using the earlier argument, for the impact at $\mathrm{C}$, the instantaneous impulse value $P_{C}$ should be chosen as the independent variable. But equation (8) shows that the value of $P_{C}$ directly depends on the value of $P_{I}$. Hence for the impact at $\mathrm{C}$ also, $P_{I}$ could still be considered as the 
independent variable. This makes it possible to have $P_{I}$ as the independent variable for all the impulse forces involved in this analysis.

For the impact at $\mathrm{C}$, the slip takes place on the XY plane. Let $s^{\prime}$ be the slip speed, and $\Phi^{\prime}$ be the direction of slip measured from the $\mathrm{X}$ axis. Now, the components along $\mathrm{X}$ and $\mathrm{Y}$ directions are,

$\dot{x}_{C}=s^{\prime}\left(P_{I}\right) \cos \left(\Phi^{\prime}\left(P_{I}\right)\right)$

$\dot{y}_{C}=s^{\prime}\left(P_{I}\right) \sin \left(\Phi^{\prime}\left(P_{I}\right)\right)$

Hereafter the independent variable $P_{I}$ is omitted from all equations for the sake of simplicity. When $s^{\prime}>0$, the impulse forces along $\mathrm{X}$ and $\mathrm{Y}$ directions, also using equation (8), can be written as

$$
\begin{aligned}
\Delta P_{x}^{C} & =-\mu_{s} \cos \Phi^{\prime} \Delta P_{C} \\
& =-\mu_{s} \cos \Phi^{\prime}\left(\sin \theta+\mu_{w} \sin \Phi \cos \theta\right) \Delta P_{I} \\
\Delta P_{y}^{C} & =-\mu_{s} \sin \Phi^{\prime} \Delta P_{C} \\
& =-\mu_{s} \sin \Phi^{\prime}\left(\sin \theta+\mu_{w} \sin \Phi \cos \theta\right) \Delta P_{I}
\end{aligned}
$$

Where $\mu_{s}$ is the coefficient of friction between the ball and table surface.

\subsection{Velocity relationships}

The velocity of any point on sphere's surface could be written, in vectorial notation, as

$V=V_{G}+\omega \Lambda R$

Hence,

$\Delta V=\Delta V_{G}+\Delta \omega \Lambda R$

From (11), resolving components along the axes appropriately, slip velocities along any axis can be expressed in terms of the centroid velocities of the ball.

At point I,

$\Delta \dot{x}_{I}=\Delta \dot{x}_{G}+\Delta \omega_{y} R \sin \theta-\Delta \omega_{z} R \cos \theta$ 
$\Delta \dot{y}_{I}^{\prime}=-\Delta \dot{y}_{G} \sin \theta+\Delta \dot{z}_{G} \cos \theta+\Delta \omega_{x} R$

At point $\mathrm{C}$, along the $\mathrm{X}$ axis,

$\Delta \dot{x}_{C}=\Delta \dot{x}_{G}-\Delta \omega_{y} R$

Along the $\mathrm{Y}$ axis,

$\Delta \dot{y}_{C}=\Delta \dot{y}_{G}+\Delta \omega_{x} R$

In equation (3a), when substituting the expression for $\Delta P_{x}^{I}$ and $\Delta P_{x}^{C}$ from equations (7a) and (10a), $\Delta \dot{x}_{G}$, which is the increment in the centroid velocity in the $\mathrm{X}$ direction $\dot{x}_{G}$, can be expressed in terms of all the instantaneous velocities by,

$\Delta \dot{x}_{G}=-\frac{1}{M}\left[\mu_{w} \cos \Phi+\mu_{s} \cos \Phi^{\prime}\left(\sin \theta+\mu_{w} \sin \Phi \cos \theta\right)\right] \Delta P_{I}$

When $\Delta P_{I} \rightarrow 0$ this equation could be written as,

$\frac{d \dot{x}_{G}}{d P_{I}}=-\frac{1}{M}\left[\mu_{w} \cos \Phi+\mu_{s} \cos \Phi^{\prime}\left(\sin \theta+\mu_{w} \sin \Phi \cos \theta\right)\right]$

The differential equation in (14a) together with other 5 similar equations for centroid velocities $\left\{\dot{y}_{G}, \ldots ., \omega_{z}\right\}$ describe the motion of the ball completely. An analytical solution does not exist. However, a numerical solution is possible.

\section{A NUMERICAL SOLUTION}

As seen earlier the solution warrants a numerical solution. The numerical solution requires initial conditions, the conditions for motion transitions like sliding to rolling, and numerical values for the parameters involved in the equations such as $\mu_{s}$.

\subsection{Initial conditions}

Referring to Fig. 2, the initial conditions for the centroid velocities of the ball are, 
$\left(\dot{x}_{G}\right)_{1}=V_{0} \cos \alpha,\left(\dot{y}_{G}\right)_{1}=V_{0} \sin \alpha,\left(\dot{z}_{G}\right)_{1}=0,\left(\omega_{x}\right)_{1}=-\omega_{0}^{S} \sin \alpha,\left(\omega_{y}\right)_{1}=\omega_{0}^{S} \cos \alpha$, and $\left(\omega_{z}\right)_{1}=\omega_{0}^{T}$,

and the initial slip speeds,

$$
\begin{aligned}
& s(0)=\left|\sqrt{\left[V_{0} \cos \alpha+R\left(\omega_{0}^{T} \cos \alpha \sin \theta-\omega_{0}^{S} \cos \theta\right)\right]^{2}+\left[-V_{0} \sin \alpha \sin \theta-R \omega_{0}^{T} \sin \alpha\right]^{2}}\right| \\
& s^{\prime}(0)=\left|V_{0}-R \omega_{0}^{T}\right| .
\end{aligned}
$$

\subsection{Friction coefficients and the condition for rolling}

When rolling occurs slip speed $s$ (or $s^{\prime}$ for the sliding on table-felt), becomes zero. At this instance the relative motion between bodies stop at their contact point along the common tangent; the friction forces become zero.

1) When $s=0$, the ball will be rolling on the cushion at the contact point I.

$$
\Delta P_{x}^{I}=\Delta P_{y^{\prime}}^{I}=0 \text {, and from (3c), } \Delta P_{C}=0 \text {. Hence, } \Delta P_{x}^{C}=\Delta P_{y}^{C}=0
$$

2) When $s^{\prime}=0$, the ball will roll on the table surface, and

$$
\Delta P_{x}^{C}=\Delta P_{y}^{C}=0
$$

In a previous work by these authors, high-speed camera based measurements were used to determine the sliding coefficient of friction for a snooker ball and table-felt; the sliding coefficient of friction, $\mu_{s}$, was found to be between 0.178-0.245 [14]. Marlow [15] suggests a value of 0.2 for pool. Since the authors of this work have obtained an experimental plot for the cushion-ball impact in snooker, parameter values found in snooker could be easily obtained [14]. Hence here onwards the numerical values found in snooker are used for the calculations. $\mu_{s}$ is taken as 0.212 , as an average value. In snooker, $\mathrm{M}=0.1406 \mathrm{~kg}, \mathrm{R}=26.25$ $\mathrm{mm}$. 


\subsection{The coefficient of restitution and impact mechanics}

According to Stronge [13], the energetic coefficient of restitution $e_{e}$, is independent of friction and the process of slip. $e_{e}^{2}$ is the negative of the ratio of the work done by the impulse force during the restitution phase to that during the compression phase. Work done at the contact point I, along the axis $Z^{\prime}$, is

$\Delta W_{Z_{I}^{\prime}}=\int_{t}^{t+\Delta t} F_{I} \cdot \dot{z}^{\prime}{ }_{I} d t=\int_{P_{I}}^{P_{I}+\Delta P_{I}} \dot{z}_{I}{ }_{I} d P_{I}$

The equivalent numerical form is,

$\left(W_{Z_{I}^{\prime}}\right)_{n+1}-\left(W_{Z_{I}^{\prime}}\right)_{n}=\Delta P_{I} \frac{\left[\left(\dot{z}_{I}^{\prime}\right)_{n+1}+\left(\dot{z}_{I}^{\prime}\right)_{n}\right]}{2}$

Where $\dot{z}_{I}^{\prime}$ is the relative velocity in the direction of the common normal at the contact point I (here it is assumed that the cushion did not move large enough to affect a change in the relative velocity i.e. the cushion is treated as a rigid body), and $F_{I}$ is the normal force from the cushion on the ball. When $P_{I}^{f}, P_{I}^{c}$ denote the accumulated impulse at the termination of impulse, and at the termination of compression, respectively, it could be shown that [13],

$e_{e}{ }^{2}=\frac{-\int_{P_{I}^{c}}^{P_{I}^{f}} \dot{z}_{I}^{\prime} d P_{I}}{\int_{0}^{P_{I}^{c}} \dot{z}^{\prime}{ }_{I} d P_{I}}$

This is rearranged as,

$W_{Z_{I}^{\prime}}\left(P_{I}^{f}\right)=\left(1-e_{e}^{2}\right) W_{Z_{I}^{\prime}}\left(P_{I}^{c}\right)$

The termination of compression occurs when the normal component of relative velocity becomes zero, i.e.

$\dot{z}_{I}^{\prime}\left(P_{I}^{c}\right)=0$

According to Marlow [15], the coefficient of restitution between the cushion and ball $e_{e}$, is 0.55 for pool. However, the authors of this work have obtained an experimental plot for the 
ball-cushion impact in snooker, where a snooker ball, under the conditions of rolling $\left(\omega^{T}{ }_{0}=\right.$ $\left.V_{0} / R\right)$ and no sidespin $\left(\omega^{S}{ }_{0}=0\right)$, was shot to collide with the cushion perpendicularly $(\alpha=0)$, and tracked using a machine vision camera [14]. The incident vs. rebound speed plot obtained was used to conclude that the equivalent coefficient of restitution, for a rolling ball perpendicularly colliding with the cushion, has a value of 0.818 , on average; the experimental plot is shown in Fig. 5. However, it should be noted that this value of 0.818 incorporates the effects of friction and the 3-dimensionality of the impulse configuration, and only stands as a representative value for the coefficient of restitution.

\subsection{The numerical algorithm}

The equation (14a) and 5 other differential equations could be written in numerical form as,

$$
\left(\dot{x}_{G}\right)_{n+1}-\left(\dot{x}_{G}\right)_{n}=-\frac{1}{M}\left\{\mu_{w} \cos (\Phi)_{n}+\mu_{s} \cos \left(\Phi^{\prime}\right)_{n}\left[\sin \theta+\mu_{w} \sin (\Phi)_{n} \cos \theta\right]\right\} \Delta P_{I}
$$

where, using equation sets (12) and (13),

$$
\tan (\Phi)_{n}=\frac{-\left(\dot{y}_{G}\right)_{n} \sin \theta+\left(\dot{z}_{G}\right)_{n} \cos \theta+\left(\omega_{x}\right)_{n} R}{\left(\dot{x}_{G}\right)_{n}+\left(\omega_{y}\right)_{n} R \sin \theta-\left(\omega_{z}\right)_{n} R \cos \theta}
$$

and $\tan \left(\Phi^{\prime}\right)_{n}=\frac{\dot{y}_{G}+\omega_{x} R}{\dot{x}_{G}-\omega_{y} R}$

The numerical scheme is written in MATLAB $^{\circledR}$ programming language. The values of $V_{0}$, $\omega_{0}^{T}, \omega_{0}^{S}, \alpha$ are the inputs to the scheme. The smaller the value of the increment in impulse $P_{I}$, i.e. $\Delta P_{I}$, in (15a), the more accurate the results will be. The aim is to find the centroid velocities of the balls at the final accumulated impulse value $P_{I}^{f}$.

The algorithm starts by calculating the increments in the centroid velocities of both balls by using equation (15a) and 5 other simultaneous equations. Using these and equations (12a), (12b), (13a) and (13b) the new slip velocities are calculated. The code is designed to 
incorporate the necessary modifications necessary, when a rolling condition is reached at either of the sliding contacts, as given in equations (16a) and (16b). The values of all physical parameters involved in the dynamics are saved as arrays, including the work done at I along the $Z^{\prime}$ axis, $W_{Z_{I}^{\prime}}$, calculated from (17a). The latest parameter values are appended to these arrays once each $\Delta P_{I}$ is applied.

Again $P_{I}^{f}$ can not be found analytically and has to be obtained numerically using the equations (17a) and (17b). The numerical scheme is initially be stopped when $\dot{z}_{I}^{\prime}=0$ (i.e. when the compression phase has ended), and the corresponding value of work done is obtained from the array containing $W_{Z_{I}^{\prime}}$, which will be $W_{Z_{I}^{\prime}}\left(P_{I}^{c}\right)$. Now, using (17b), the value $W_{Z_{I}^{\prime}}\left(P_{I}^{f}\right)$ can be calculated, given that $e_{e}$ is known. Now the numerical process can resume and when $W_{Z_{I}^{\prime}}=W_{Z_{I}^{\prime}}\left(P_{I}^{f}\right)$, the numerical process is terminated. The rebound velocity values of the ball centroid are the last entries in the arrays of the respective velocity components.

Note: In order to assume a reasonable value for $\Delta P_{I}$ to start the numerical scheme with, an approximate value for $P_{I}^{f}$ could be taken as $\left(1+e_{e}\right) M V_{0} \sin \alpha$, the value of the normal impulse to the cushion, had the ball without spinning collided into a solid flat wall. Approximately for $N$ iterations, $\Delta P_{I}=\frac{\left(1+e_{e}\right) M V_{0} \sin \alpha}{N}$. Obviously the values of $P_{I}^{c}$ and $P_{I}^{f}$ will decide the actual number of iterations that have taken place in the scheme. An initial $N$ of 5000 worked satisfactorily for the scheme.

\subsection{Estimating $e_{e}$ and $\mu_{w}$}

The experimental plot in Fig. 5 was obtained under the conditions of $\omega^{S}{ }_{0}=0, \alpha=0$, and $\omega^{T}{ }_{0}=$ $V_{0} / R$, on a Riley® Renaissance type snooker table that is also the official table brand of the 
World Snooker Association and is used in all its professional tournaments. It is known that $0<e_{e} \leq 1$. For each of the experimentally obtained incident speed values (i.e. $V_{0}$ ) in the speed range of $V_{0}<1.5 \mathrm{~m} / \mathrm{s}$, the numerical algorithm was run for values of $e_{e}$ starting from 0 to 1 in the increments of 0.01 , and the rebound speed $\dot{y}_{G}\left(P_{I}^{f}\right)$ was obtained; higher speeds were not considered as the assumption of rigid cushion may not be applicable for those values. The value of $e_{e}$ that minimises the RMS value of all errors between the experimental and numerically predicted rebound speeds should be the actual value for the coefficient of restitution between the cushion and ball. Calculations showed, that $e_{e}=0.98$ and $\mu_{w}=0.14$.

Numerically obtained rebound speed values for $e_{e}=0.98$ and $\mu_{w}=0.14$, are plotted in Fig. 5 together with experimentally obtained values. As seen in Fig. 5(b), numerically obtained values of incident speed deviate from the experimentally obtained values for speeds $V_{0}>2.5$ $\mathrm{m} / \mathrm{s}$. Quite possibly, this could be the velocity limit beyond which the no deformation assumption for cushion would not be valid anymore. But $V_{0}=2.5 \mathrm{~m} / \mathrm{s}$ is a considerably high speed as far as snooker is concerned. For oblique shots, the ones for which the normal component of incident velocity of less than $2.5 \mathrm{~m} / \mathrm{s}$, would only be analysed using the numerical algorithm described in 3.4.
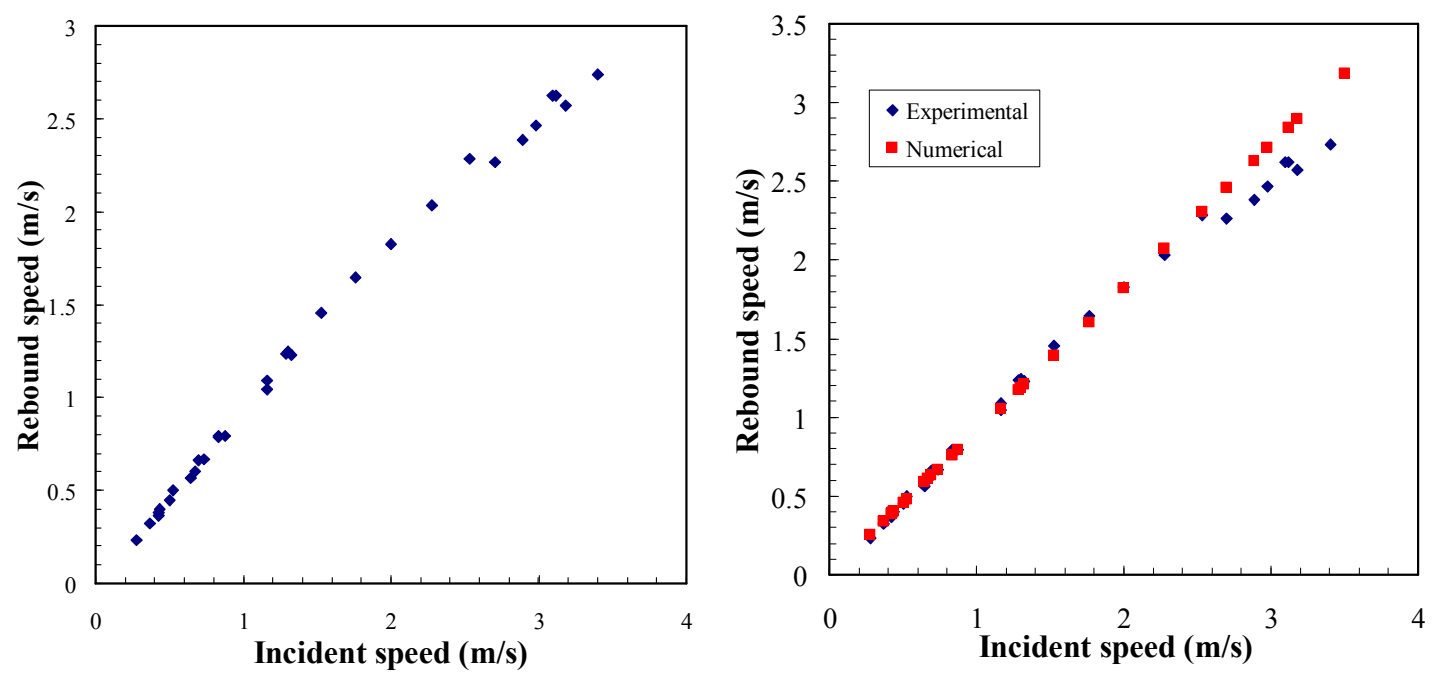

Fig. 5 Rebound speed versus incident velocity, obtained experimentally and numerically 


\section{RESULTS AND DISCUSSION}

The results obtained from the numerical algorithm are given in Fig. 6, 7, and 8. Once the ball is struck by the cue stick the ball generally slides, where $\omega_{0}^{T} \neq V_{0} / R$, but quickly the rolling condition of $\omega_{0}^{T}=V_{0} / R$ is brought about by the action of the friction between the ball and table (an interested reader could see the plots given in [14]). Hence the balls before they impact the cushions in billiards are generally in the rolling state, possibly with some sidespin.

The simplest case of rolling with no sidespin is shown in Fig. 6. The plot of rebound speed versus incident angle for different incident ball speeds tells that the rebound speed increases with the incident speed. The second plot in Fig. 6, suggests that the rebound angle is only influenced by the incident angle, for a rolling ball with no sidespin prior to the impact.
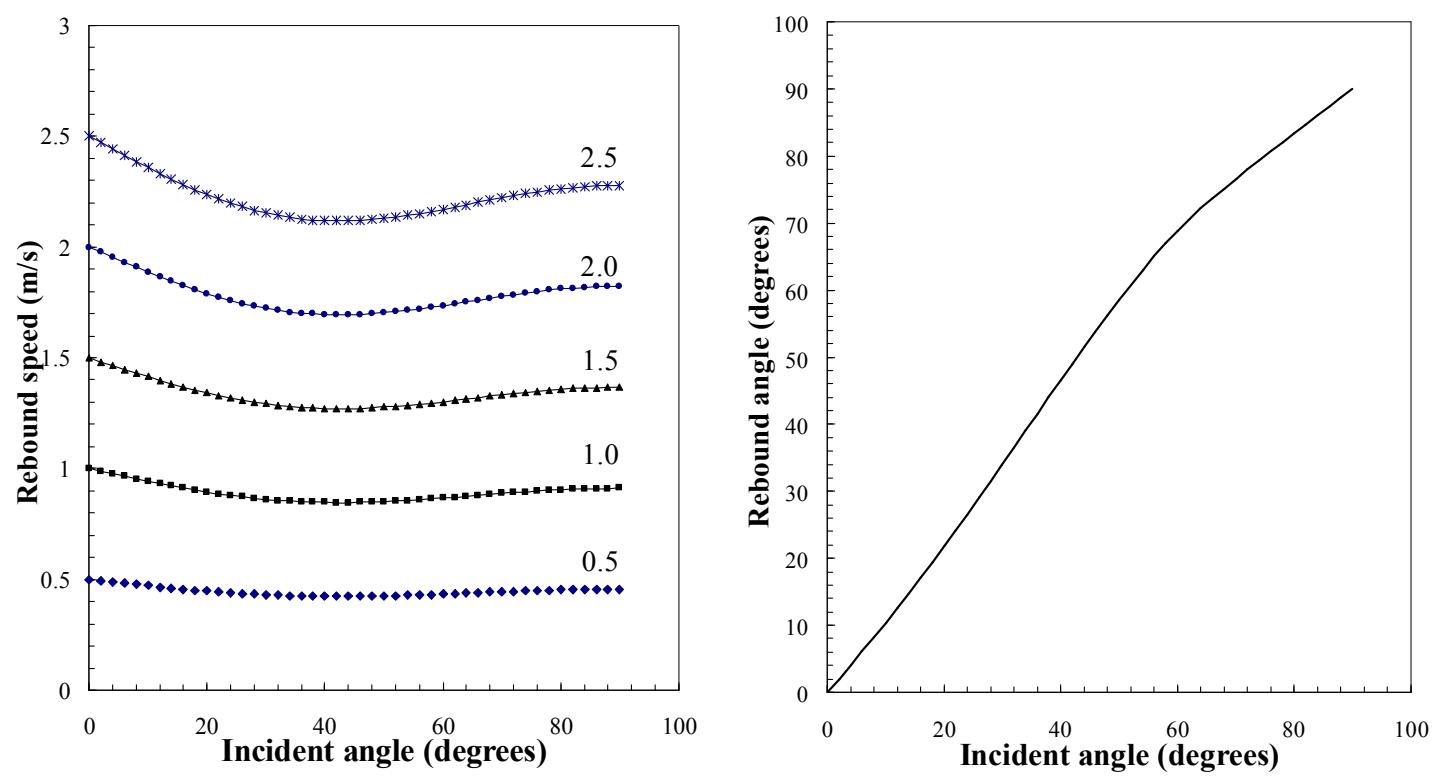

Fig. 6 Rebound speed and rebound angle versus incident angle for different rolling velocities with no sidespin $\left(\omega^{T}{ }_{0}=V_{0} / R, \omega^{S}=0\right)$ 
According to Fig. 7, the amount of top spin a ball has affects the ball rebound speed by up to $15 \%$. The speed loss is the largest for ball incident angles around $30^{\circ}$. For over-spinning ball the speed loss is generally low. The rebound angles are not greatly affected by the topspin of the ball as seen in the second plot of Fig. 7.
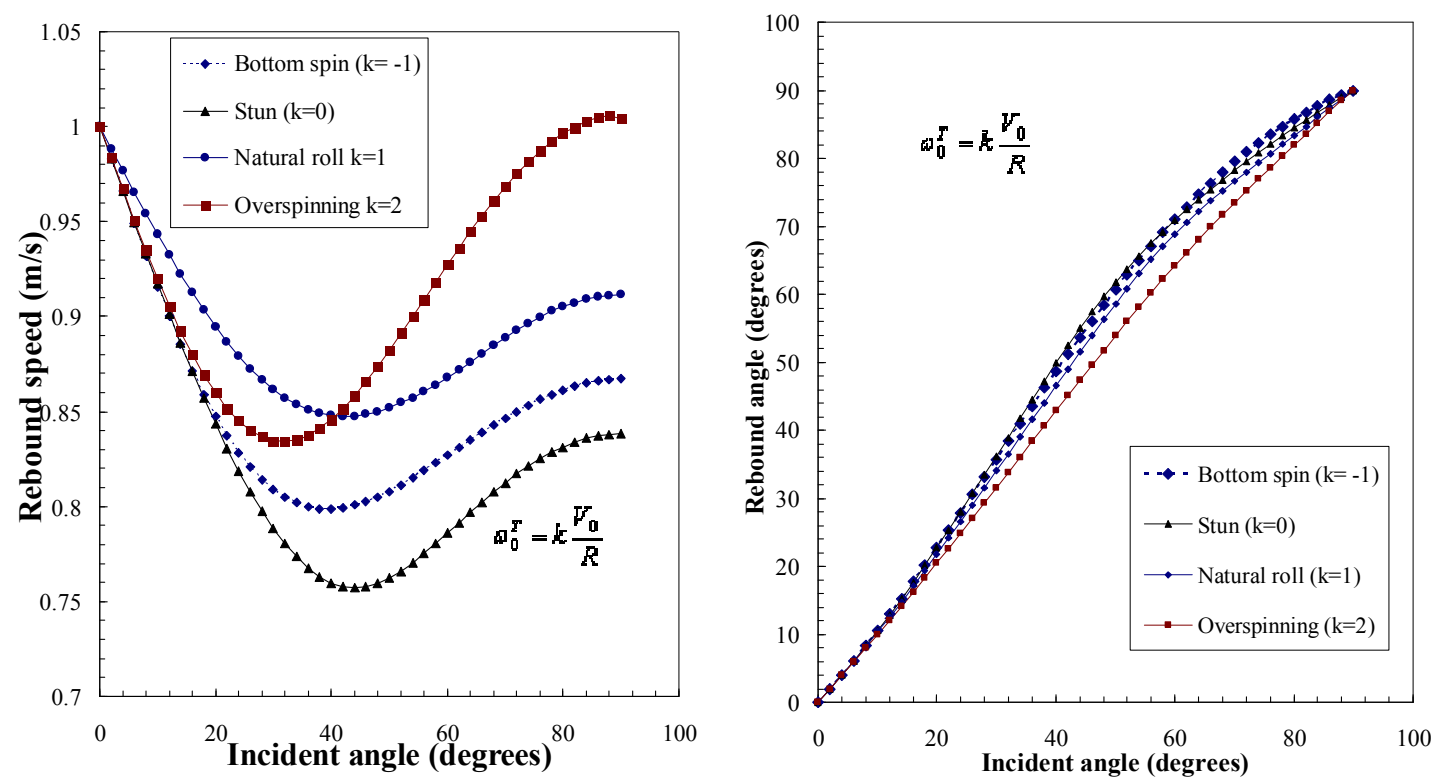

Fig. 7 Rebound speed and rebound angle versus incident angle for different topspins of the ball, $\omega^{T}{ }_{0}=k V_{0} / R$, and $V_{0}=1 \mathrm{~m} / \mathrm{s}$ with no sidespin $\left(\omega^{S}{ }_{0}=0\right)$
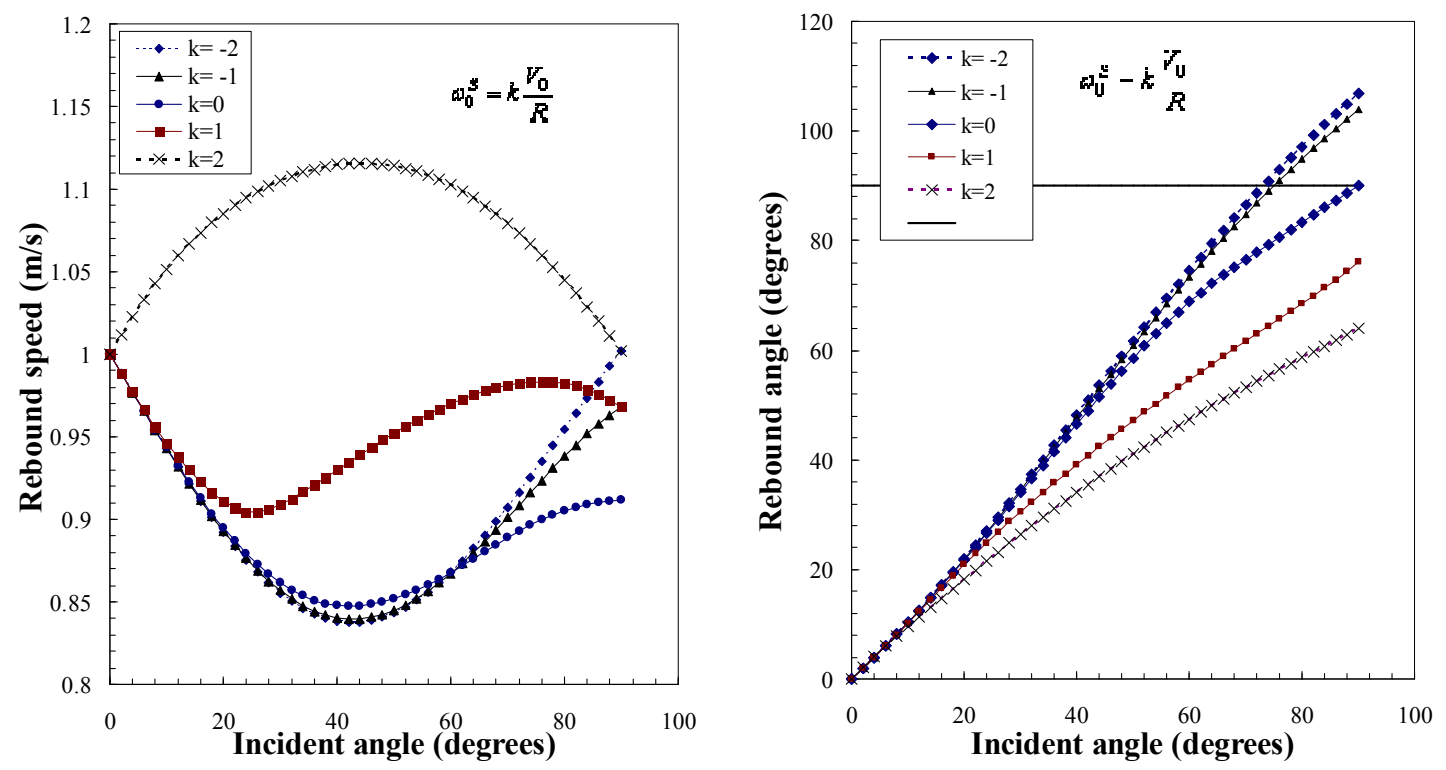

Fig. 8 Rebound speed and rebound angle versus incident angle for different sidespins of the ball, $\omega^{S}{ }_{0}=k V_{0} / R$, and $V_{0}=1 \mathrm{~m} / \mathrm{s}$ with the ball rolling $\left(\omega^{T}{ }_{0}=V_{0} / R\right)$ 
The Fig. 8 presents some very interesting results. When the ball has right spin (according to billiards terminology, $\omega^{S}{ }_{0}$ as marked in Fig. 2 is called right spin, the opposite of which is left spin), the rebound speed the exceeds that value of the incident speed. Also for higher values of left spin, at higher incident angles towards $90^{\circ}$, the rebound velocity exceeds the value of the incident ball speed. The second plot in Fig. 8 suggest that when the ball has left spin $(\mathrm{k}<0)$, and for incident angles values close to $90^{\circ}$, the ball bounces back to the side that it approached the cushion from (see Fig. 9). This effect has been described by Walker [16] for billiards, and by Cross [17] in a general context for the bounce of ball. Cross [17] also presents some experimental values for a tennis ball bouncing on a rough surface.

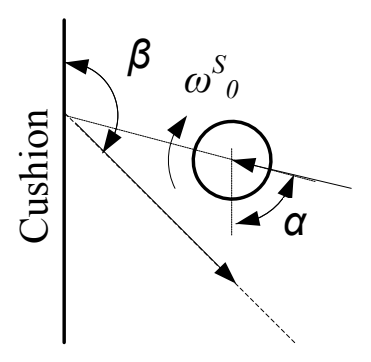

Fig. 9 Ball bouncing back to the same side under left spin conditions for $\alpha$ 's close to $90^{\circ}$

A plot of sliding speeds against the instantaneous impulse value is shown in Fig. 10. The change in slip directions as indicated by the plot suggests that the assumption of unidirectional slip can not be true. 


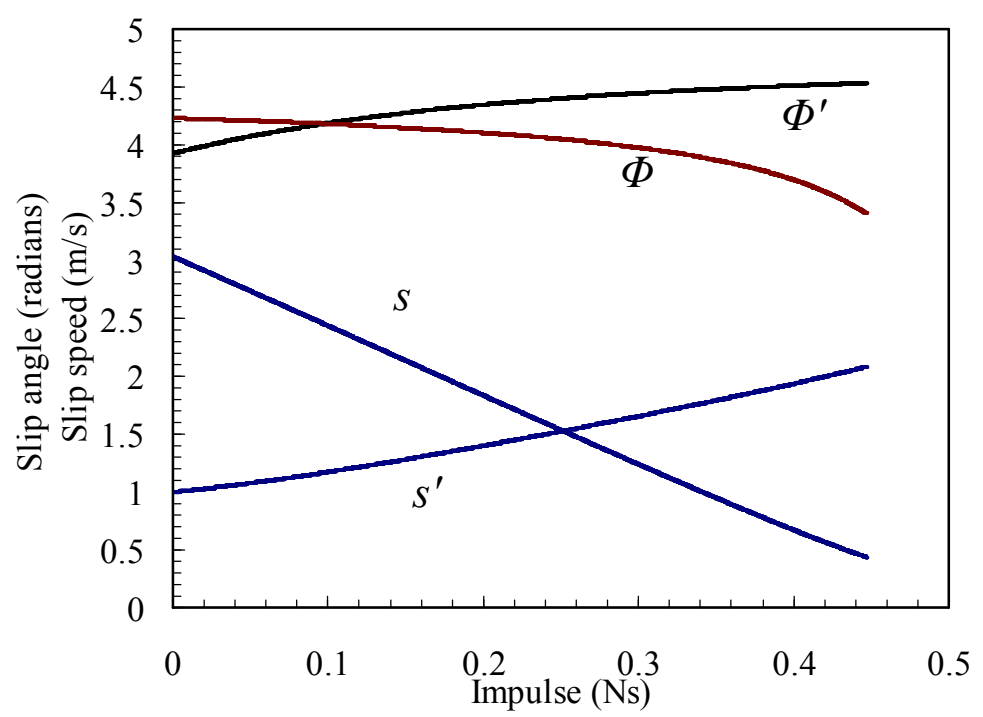

Fig. 10 Slip-impulse curves for $V_{0}=2 \mathrm{~m} / \mathrm{s}, \alpha=45^{\circ}, \omega^{S}{ }_{0}=2 V_{0} / R$ and $\omega^{T}{ }_{0}=1.5 V_{0} / R$ ( $s$ and $\Phi$ are for the slip at the cushion, and $s^{\prime}$ and $\Phi^{\prime}$ are for the slip at the table )

\section{CONCLUSIONS}

A 3-dimensional impact analysis for the collision of a spinning billiard ball into a cushion is presented. Differential equations are derived for ball dynamics during the time of impact and then the solutions are found numerically.

Combining some previous experimental results from the authors and the numerical solutions the coefficient of restitution for the ball-cushion collision is determined as 0.98 . In the same way, the value for the sliding coefficient of friction is found to be 0.14 .

The rebound angles and speeds are given as plots against the incident angles and speed for different velocity and spin conditions. Under excessive sidespin conditions the rebound speeds are found to exceed the incident speeds. Under these conditions the ball is also found to bounce back on the same side as it approached the cushion.

Although this analysis provides the quantification for many phenomena involved with cushion collisions that are described in billiards related literature, it is expected to be 
validated by tracking spin of a billiard ball. A colour pattern drawn on a white Cue ball may be used for this purpose.

\section{REFERENCES}

1 Nadler, D. Mathematical Theory of Spin, Friction, and Collision in the Game of Billiards, 2005 (David Nadler). An English translation of Coriolis' 1835 book.

2 Long, F., Herland, J, Tessier, M.-C., Naulls, D., Roth, A., Roth, G., and Greenspan, M. Robotic Pool: An Experiment in Automatic Potting. In IROS'04: IEEE/RSJ International Conference on Intelligent Robotics and Systems, Sendai, Japan, 2004, vol. 3, pp. 25202525 .

3 Ho, K. H. L., Martin, T., and Baldwin, J. Snooker Robot Player - 20 Years on. In IEEE Symposium on Computational Intelligence and Games, 2007 (CIG 2007), Hawaii, 1-5 April 2007, pp.1-8.

4 Cheng, B.-R., Li., J.-T, and Yang, J-S. Design of the Neural-Fuzzy Compensator for a Billiard Robot. In 2004 IEEE International Conference on Networking, Sensing \& Control, Taipei, Taiwan, 21-23 March 2004, pp. 909-913.

5 Alian, M. E., Shouraki, S. E., Shalmani, M. T. M., Karimian, P., and Sabzmeydani, P. Roboshark: A Gantry Pool Player. 35th International Symposium on Robotics (ISR), Paris, France, 2004.

6 Jebara, T., Eyster, C., Weaver, J., Starner, T., and Pentland, A. Stochasticks: Augmenting the Billiards Experience with Probabilistic Vision and Wearable Computer. In Proceedings of the IEEE International Symposium on Wearable Computers, Cambridge, MA, USA, Oct 1997, pp. 138-145. 
7 Larsen, L. B., Jensen, M. D., and K, V. W. (2002). Multi Modal User Interaction in an Automatic Pool Trainer. In Proceedings of the Fourth IEEE International Conference on Multimodal Interfaces (ICMI'02), Pittsburgh, USA, 14-16 Oct 2002 pp. 361-366.

8 Smith, M. PickPocket: A computer billiards shark. Artificial Intelligence, 2007, 171(1617), pp. 1069-1091.

9 Dussault, J.-P., and Landry, J.-F. Optimization of a Billiard Player - Tactical Play. LECTURE NOTES IN COMPUTER SCIENCE, 2007, 4630, pp. 256-270.

10 Leckie, W., and Greenspan, M. An Event-Based Pool Physics Simulator. LECTURE NOTES IN COMPUTER SCIENCE, 2006, 4250, pp. 247-262.

11 Marlow, W. C. The Physics of Pocket Billiards, 1994 (MAST, Florida, USA).

12 Alciatore, D. G. Pool and Billiards Physics Principles by Coriolis and Others, submitted to the Am. J. Phys., Retrieved on 20 January 2009, from http://billiards.colostate.edu/physics/Alciatore_AJP_MS22090_revised_pool_physics_arti cle.pdf

13 de la Torre Juárez, M. The Effect of Impulsive Forces on a System with Friction: The Example of the Billiard Game. European Journal of Physics, 1994, 15(4), pp. 184-190.

14 Stronge, W. J. Impact Mechanics, 2000, (Cambridge University Press, Cambridge, UK)

15 Mathavan, S., Jackson, M. R., and Parkin, R. M. Application of high-speed imaging in determining the dynamics involved in Billiards, American Journal of Physics, 2009, $\mathrm{XX}(\mathrm{X}), \mathrm{pp} . \mathrm{XXX}$

16 Walker, J. The Physics of the Follow, the Draw and the Masse (in Billiards and Pool). Scientific American, 1983, 249, pp. 124-129.

17 Cross, R. Bounce of a spinning ball near normal incidence, American Journal of Physics, 2005, 73(10), 914-92.

\section{APPENDIX 1}




\section{Notation}

$e_{e}$ coefficient of restitution between the ball and cushion

$F$ force

I moment of inertia of the ball

$M$ mass of the ball

$N$ Number of iterations

$P \quad$ accumulated impulse at any time during impact

$P_{I}^{c}$ accumulated impulse at the termination of compression

$P_{I}^{f}$ the final accumulated value of impulse

$R$ radius of the ball

$s \quad$ slip speed

$V_{0}$ incident speed of the ball

$W$ work done due to impulse force

$\alpha \quad$ ball incident angle with cushion

$\beta$ rebound angle

$\Delta P$ impulse during a time of $\Delta t$

$\theta$ the angle that the common normal of the ball-cushion contact point makes with the horizontal

$\mu_{s}$ coefficient of sliding friction between the ball and table

$\mu_{w}$ coefficient of sliding friction between the ball and cushion

$\Phi$ slip direction

$\omega$ angular velocity of the ball

$\omega^{T}{ }_{0}$ top spin of the ball at incidence

$\omega^{S}$ side spin of the ball at incidence 